\title{
SPACES WHICH INVERT WEAK HOMOTOPY EQUIVALENCES
}

\author{
JONATHAN ARIEL BARMAK ${ }^{\dagger}$
}

\begin{abstract}
It is well known that if $X$ is a CW-complex, then for every weak homotopy equivalence $f: A \rightarrow B$, the map $f_{*}:[X, A] \rightarrow[X, B]$ induced in homotopy classes is a bijection. For which spaces $X$ is $f^{*}:[B, X] \rightarrow[A, X]$ a bijection for every weak equivalence $f$ ? This question was considered by J. Strom and T. Goodwillie. In this note we prove that a non-empty space inverts weak equivalences if and only if it is contractible.
\end{abstract}

We say that a space $X$ inverts weak homotopy equivalences if the functor $[-, X]$ inverts weak equivalences, that is, for every weak homotopy equivalence $f: A \rightarrow B$, the induced map $f^{*}:[B, X] \rightarrow[A, X]$ is a bijection. As usual $[A, X]$ stands for the set of homotopy classes of maps from $A$ to $X$. This property is clearly a homotopy invariant. In [1] Jeff Strom asked for the characterization of such spaces. Tom Goodwillie observed that if $X$ inverts weak equivalences and is $T_{1}$ (i.e. its points are closed), then each path-component is weakly contractible (has trivial homotopy groups) and then contractible. His idea was to use finite spaces weak homotopy equivalent to spheres. A map from a connected finite space to a $T_{1}$-space has a connected and discrete image and is therefore constant. This is one of the many interesting applications of non-Hausdorff spaces to homotopy theory. Goodwillie also proved that if a space inverts weak equivalences, then it must be connected. In this note we follow his ideas and give a further application of non-Hausdorff spaces to obtain the expected characterization:

Theorem 1. A non-empty space $X$ inverts weak homotopy equivalences if and only if it is contractible.

Lemma 2 (Goodwillie). Suppose that $X$ inverts weak homotopy equivalences and is weakly contractible. Then it is contractible.

Proof. Just take the weak homotopy equivalence $X \rightarrow *$.

Proposition 3 (Goodwillie). Let $X$ be a space which inverts weak homotopy equivalences. Then it is connected.

Proof. We can assume $X$ is non-empty. Suppose that $X_{0}$ and $X_{1}$ are two path-components of $X$. Let $x_{0} \in X_{0}$ and $x_{1} \in X_{1}$. Let $A=\mathbb{N}_{0}$ be the set of nonnegative integers with the discrete topology and $B=\{0\} \cup\left\{\frac{1}{n}\right\}_{n \in \mathbb{N}} \subseteq \mathbb{R}$ with the usual topology. The map $f: A \rightarrow B$ which maps 0 to 0 and $n$ to $\frac{1}{n}$ for every $n$, is a weak homotopy equivalence. Take $g: A \rightarrow X$ defined by $g(0)=x_{0}$ and $g(n)=x_{1}$ for every $n \geq 1$. By hypothesis there exists a map $h: B \rightarrow X$ such that $h(0) \in X_{0}$ and $h\left(\frac{1}{n}\right) \in X_{1}$ for every $n \geq 1$. Since $\frac{1}{n} \rightarrow 0$, $X_{0}$ intersects the closure of $X_{1}$. Thus $X_{0}$ and $X_{1}$ are contained in the same component of $X$.

2010 Mathematics Subject Classification. 55Q05, 55P15, 54G05, 54G10.

Key words and phrases. Weak homotopy equivalences, homotopy types, non-Hausdorff spaces.

$\dagger$ Researcher of CONICET. Partially supported by grant UBACyT 20020160100081BA. 
Lemma 4. Let $X$ be a space which inverts weak equivalences and let $Y$ be a locally compact Hausdorff space. Then the mapping space $X^{Y}$, considered with the compact-open topology, also inverts weak equivalences.

Proof. This follows from a direct application of the exponential law and the fact that a weak equivalence $f: A \rightarrow B$ induces a weak equivalence $f \times 1_{Y}: A \times Y \rightarrow B \times Y$.

By Lemmas 2 and 4 it only remains to show that a map that inverts weak equivalences is path-connected. If we require a slightly different property, this is easy to prove using only Hausdorff spaces. The following result is not needed for the proof of Theorem 1 .

Proposition 5. Let $\left(X, x_{0}\right)$ be a pointed space such that for every weak homotopy equivalence $f: A \rightarrow B$ between Hausdorff spaces and every $a_{0} \in A$, the induced map

$$
f^{*}:\left[\left(B, f\left(a_{0}\right)\right),\left(X, x_{0}\right)\right] \rightarrow\left[\left(A, a_{0}\right),\left(X, x_{0}\right)\right]
$$

is a bijection. Then $X$ is path-connected.

Proof. Let $X_{0}$ be the path-component of $x_{0}$. Let $X_{1}$ be any path-component of $X$ and let $x_{1} \in X_{1}$. Let $A, B, f, g$ be as in Proposition 3, Let $a_{0}=0 \in A$. By hypothesis there exists $h:(B, 0) \rightarrow\left(X, x_{0}\right)$ such that $h f \simeq g$ rel $\{0\}$. In particular $h(1) \in X_{1}$. Define $h^{\prime}: B \rightarrow X$ by $h^{\prime}(0)=x_{0}$ and $h^{\prime}\left(\frac{1}{n}\right)=h\left(\frac{1}{n+1}\right)$ for $n \geq 1$. The continuity of $h^{\prime}$ follows from that of $h$. Since $h^{\prime}\left(\frac{1}{n}\right)=h\left(\frac{1}{n+1}\right) \in X_{1}$ and $h\left(\frac{1}{n}\right) \in X_{1}$ for every $n \geq 1$, there exists a homotopy $H: A \times I \rightarrow Y$ from $f^{*}\left(h^{\prime}\right)$ to $f^{*}(h)$. Moreover we can take $H$ to be stationary on $0 \in A$. Since $f^{*}:\left[(B, 0),\left(X, x_{0}\right)\right] \rightarrow\left[(A, 0),\left(X, x_{0}\right)\right]$ is injective, there exists a homotopy $F: B \times I \rightarrow X, F: h^{\prime} \simeq h$ rel $\{0\}$. The map $F$ gives a collection of paths from $h\left(\frac{1}{n+1}\right)$ to $h\left(\frac{1}{n}\right)$. We glue all these paths to form a path from $x_{0}$ to $h(1)$. That is, define $\gamma: I \rightarrow X$ by $\gamma(0)=x_{0}$ and $\gamma(t)=F\left(\frac{1}{n},\left(\frac{1}{n}-\frac{1}{n+1}\right)^{-1}\left(t-\frac{1}{n+1}\right)\right)$ if $t \in\left[\frac{1}{n+1}, \frac{1}{n}\right]$. Note that $\gamma$ is continuous in $t=0$ for if $U \subseteq X$ is a neighborhood of $x_{0}$, then $\{0\} \times I \subseteq F^{-1}(U)$, and by the tube lemma there exists $n_{0} \geq 1$ such that $\left\{\frac{1}{n}\right\} \times I \subseteq F^{-1}(U)$ for every $n \geq n_{0}$. Then $\left[0, \frac{1}{n_{0}}\right] \subseteq \gamma^{-1}(U)$. Hence, $x_{0}$ and $h(1)$ lie in the same path-component, so $X_{0}=X_{1}$.

Note that if a contractible space $X$ satisfies the hypothesis of Proposition 5 for some point $x_{0}$, then by taking $A=B=X, a_{0}=x_{0}$ and $f$ the constant map $x_{0}$, one obtains that $\left\{x_{0}\right\}$ is a strong deformation retract of $X$. Conversely, a based space $\left(X, x_{0}\right)$ such that $\left\{x_{0}\right\}$ is a strong deformation retract of $X$, clearly satisfies the hypothesis of the proposition.

The following result is the key lemma for proving Theorem 1 and in contrast to the previous result, the proof provided uses non-Hausdorff spaces.

Lemma 6. Let $X$ be a space which inverts weak homotopy equivalences. Then $X$ is path-connected.

Proof. We can assume $X$ is non-empty. Let $X_{0}$ and $X_{1}$ be path-components of $X$. Let $B$ be a set with cardinality $\# B>\alpha=\max \{\# X, c\}$. Here $c$ denotes the cardinality $\# \mathbb{R}$ of the continuum. Consider the following topology in $B$ : a proper subset $F \subseteq B$ is closed if and only if $\# F \leq \alpha$. Note that the path-components of $B$ are the singletons, for if $\gamma: I \rightarrow B$ is a path, then its image has cardinality at most $\alpha$, so it is connected and discrete and then constant. Let $A$ be the discretization of $B$, i.e. the same set with the discrete topology. Then the identity $i d: A \rightarrow B$ is a weak homotopy equivalence. Let $b_{0}$ 
and $b_{1}$ be two different points of $B$. Define $g: A \rightarrow X$ in such a way that $g\left(b_{0}\right) \in X_{0}$ and $g\left(b_{1}\right) \in X_{1}$ (define $g$ arbitrarily in the remaining points of $A$ ). Then $g$ is continuous. Since the identity $i d^{*}:[B, X] \rightarrow[A, X]$ is surjective, there exists a map $h: B \rightarrow X$ such that $h \circ i d \simeq g$. In particular $h\left(b_{0}\right) \in X_{0}$ and $h\left(b_{1}\right) \in X_{1}$. Since $\# B>\alpha \geq \# X$ and $B=\bigcup_{x \in X} h^{-1}(x)$, there exists $x \in X$ such that $\# h^{-1}(x)>\alpha$. Let $U \subseteq X$ be an open neighborhood of $h\left(b_{0}\right)$. Then $h^{-1}\left(U^{c}\right) \subseteq B$ is a proper closed subset, so $\# h^{-1}\left(U^{c}\right) \leq \alpha$. Thus, $h^{-1}(x)$ is not contained in $h^{-1}\left(U^{c}\right)$ and then $x \in U$. Since every open neighborhood of $h\left(b_{0}\right)$ contains $x$, there is a continuous path from $x$ to $h\left(b_{0}\right)$, namely $t \mapsto x$ for $t<1$ and $1 \mapsto h\left(b_{0}\right)$. In particular $x \in X_{0}$. Symmetrically, $x \in X_{1}$. Therefore $X_{0}=X_{1}$.

Proof of Theorem 1. It is clear that a contractible space inverts weak equivalences. Suppose $X \neq \emptyset$ is a space which inverts weak equivalences. By Lemma 4, $X^{S^{n}}$ inverts weak equivalences for every $n \geq 0$ and then it is path-connected. Therefore $\pi_{n}(X)$ is trivial for every $n \geq 0$ and by Lemma $2, X$ is contractible.

\section{REFERENCES}

[1] J. Strom (https://mathoverflow.net/users/3634/jeff-strom), Spaces that invert weak homotopy equivalences., URL (version: 2010-11-23): https://mathoverflow.net/q/47042

Universidad de Buenos Aires. Facultad de Ciencias Exactas y Naturales. Departamento de Matemática. Buenos Aires, Argentina.

COniCet-Universidad de Buenos Aires. Instituto de Investigaciones Matemáticas Luis A. Santaló (IMAS). Buenos Aires, Argentina.

E-mail address: jbarmak@dm.uba.ar 